\title{
ON THE NUMBER OF SINGLE-PEAK SOLUTIONS OF THE NONLINEAR SCHRÖDINGER EQUATION is
}

\author{
Massimo GROSSI \\ Dip. di Matematica, Università di Roma "La Sapienza”, P. le A.Moro 2, 00185 Roma, Italy
}

Received 25 October 2000, revised 23 March 2001

ABSTRACT. - In this paper we consider uniqueness and multiplicity results for single-peak solutions for the nonlinear Schrödinger equation. For a suitable class of potentials $V$ and critical points $P$ we are able to establish exact multiplicity results. In particular we show that for any nondegenerate critical point of $V$ there are only one solution concentrating at $P$. Moreover some examples of single-peak solutions concentrating at the same point $P$ are provided. (C) 2002 L'Association Publications de l'Institut Henri Poincaré. Published by Elsevier B.V. All rights reserved

Keywords: Nonlinear Schrödinger equation; Nondegeneracy

RÉSUMÉ. - Dans ce papier nous considérons les résultats d'unicité et de multiplicité pour les solutions de seul-pic pour l'équation de Schrödinger non linéaire. Pour une classe convenable de potentiel $V$ et critiques points $P$ nous peuvons établir une multiplicité exacte.

(C) 2002 L'Association Publications de l'Institut Henri Poincaré. Published by Elsevier B.V. All rights reserved

\section{Introduction}

In this paper we study standing wave solutions of the nonlinear Schrödinger equation

$$
\mathrm{i} h \frac{\partial \psi}{\partial t}=-\frac{h^{2}}{2 m} \Delta \psi+V(x) \psi-|\psi|^{p-1} \psi, \quad|V(x)| \leqslant C, x \in \mathcal{R}^{N},
$$

i.e., solutions of the form

$$
\psi(x, t)=\mathrm{e}^{\mathrm{i} \frac{E t}{h}} u(x), \quad u: \mathcal{R}^{N} \rightarrow \mathcal{R}^{+} .
$$

Substituting (1.2) in (1.1) and assuming that $m=\frac{1}{2}$ one has

$$
\left\{\begin{array}{l}
-h^{2} \Delta u+(V(x)-E) u=u^{p} \\
u>0
\end{array}\right.
$$

\footnotetext{
"Supported by MURST, Project "Variational Methods and Nonlinear Differential Equation". E-mail address: grossi@mat.uniroma1.it (M. Grossi).
} 
A suitable choice of $E$ makes $V$ bounded from below by a positive constant. Hence, without loss of generality, it is possible to assume that $E=0$ and $V \geqslant V_{0}>0$. If we set $h=\varepsilon,(1.3)$ becomes

$$
\left\{\begin{array}{l}
-\varepsilon^{2} \Delta u+V(x) u=u^{p} \quad \text { in } \mathcal{R}^{N}, \\
u>0 .
\end{array}\right.
$$

In [7], Floer and Weinstein considered the case $N=1, p=3$ and they proved that for small $\varepsilon$ there exists a family of standing wave solutions which concentrates at each given nondegenerate critical point of the potential $V$. This result was generalized by Oh [16] of higher dimensions. The arguments in those papers are based on a Liapunov-Schmidt reduction.

The existence of solutions of (1.4) in the possibly degenerate setting was studied by many authors. In this context the first results seem due to Rabinowitz (see [17]) and Ding and $\mathrm{Ni}$ (see [6]). In [17] it was shown that if $\inf _{\mathcal{R}^{N}} V<\liminf _{|x| \rightarrow \infty} V(x)$ then the mountain pass theorem provides a solution for small $\varepsilon$. This solution concentrates around a global minimum of $V$ as $\varepsilon \rightarrow 0$, as shown later by Wang (see [18]).

Later Ambrosetti, Badiale and Cingolani (see [1]) obtained existence of standing wave solutions by assuming that the potential $V$ has a local minimum or maximum with nondegenerate $m$ th derivative, for some integer $m$.

Another result concerning degenerate critical points of $V$ is due to Li (see [12]), where a degeneracy of any order of the derivative is allowed. In [12] the author proves the existence of a solution for (1.4) by only assuming that the critical points of $V$ are "stable" with respect to a small $C^{1}$-perturbation of $V$. In [5] Del Pino and Felmer consider a more general nonlinearity $f(t)$ and obtained a solution of (1.4) by considering a "topologically nontrivial" critical value of the energy functional associated. In such a this way they are able to menage a wide class of critical points of $V$. We point out that all these results are obtained by suitable perturbation of the solution $U_{y}$ of

$$
\begin{cases}-\Delta u+V(y) u=u^{p} & \text { in } R^{N}, \\ u>0 & \text { in } R^{N}, \\ u(x) \rightarrow 0 & \text { as }|x| \rightarrow \infty .\end{cases}
$$

This type of solutions are sometimes called single-peak solutions since they have only one local maximum point (see Definition 2.3 for a more precise statement).

Moreover (see [18] or Theorem 2.7) concentration of single-peak solution may only occur at critical point of $V$. So the problem of the existence of at least one single-peak solution concentrating at $P$ seems to be completely described.

At this stage a question arises:

How many solutions are generated by a stable critical point $\mathrm{P}$ of $V$ ?

In this paper we try to give an answer to this question for a suitable class of potential $V$. Now we briefly describe the class of potentials $V$ considered. Roughly speaking we take in account $V \in C^{1}$ such that

$$
\frac{\partial V}{\partial x_{i}}(x)=h_{i}(x)+R_{i}(x) \text { in a neighborhood of } P,
$$


where $h_{i}$ is an homogenous function of degree $\alpha_{i}$ and $R_{i}$ is a higher order term (see Section 3 for the precise definition). In this setting the functions $h_{i}$ play an important role: indeed if we consider the following vector field

$$
\mathcal{L}_{P}(y)=\left(\int_{R^{N}} h_{i}(x+y+P) U_{P}^{2}(x) \mathrm{d} x\right)_{i=1, \ldots, N},
$$

where $U_{P}$ is the solution of (1.5) and

$$
Z=\left\{y \in \mathcal{R}^{N} \text { such that } y \text { is a stable zero of } \mathcal{L}\right\},
$$

(see Definition 3.6 for the definition of stable zero) then we get the following theorem.

THEOREM 1.1. - Let $V$ be an admissible potential (see Section 3 for the definition) and suppose that $\# Z<\infty$. If, for any $y \in Z$, it holds

$$
\operatorname{det} \operatorname{Jac} \mathcal{L}_{P}(y) \neq 0,
$$

then there exists $\varepsilon_{0}>0$ such that for any $0<\varepsilon<\varepsilon_{0}$ we have

$$
\#\{\text { single-peak solution of (1.4) concentrating at } x=P\}=\# Z \text {. }
$$

In particular it is possible to show that, if $P$ is a nondegenerate critical point of $V$ then there is only one solution which concentrates at $P$ (see Corollary 6.4). However, we think that the number of the solution of (1.4) concentrating at $P$ is influenced by the shape of $V$ in a neighborhood of $P$ instead of its nondegeneracy (see Proposition 6.3) and example at the end of Section 6). Finally, again in the example at the end of Section 6, we construct an example of a potential $V$ such that there exist exactly two single-peak solutions concentrating at the same point $P$. In the same way it is possible to give an example where a concentration of $k$ single-peak solutions at $P$ occurs. We do not know any example of such a phenomenon in literature, even for similar elliptic perturbed problems defined in bounded or unbounded domains.

Our results can be extended to the case of more general nonlinearities $f(u)$, using for example the assumption of [8]. However we state and prove our results only for the case $f(s)=s^{p}$.

The paper is organized as follows: in Section 2 we recall some known facts and introduce the main definitions. In Section 3 we introduce the class of admissible potentials $V$. In Section 4 we prove that stable zero of $\mathcal{L}_{P}$ generates solutions to (1.4). In Section 5 we establish an important estimate which allows, in Section 6, to deduce the exact multiplicity result.

\section{Known facts and main definitions}

In this section we recall some known facts. 
THEOREM 2.1. - Let $V$ a continuous function verifying $0<V_{0} \leqslant V \leqslant V_{1}$ in $\mathcal{R}^{N}$. Then for any $y \in \mathcal{R}^{N}$ the problem

$$
\begin{cases}-\Delta u+V(y) u=u^{p} & \text { in } \mathcal{R}^{N}, \\ u>0 & \text { in } \mathcal{R}^{N}, \\ u(x) \rightarrow 0 & \text { as }|x| \rightarrow \infty,\end{cases}
$$

with $1<p<\frac{N+2}{N-2}$, admits only one solution $U_{y} \in H^{1}\left(\mathcal{R}^{N}\right)$ which satisfies

(i) $U_{y}$ is spherically symmetric with respect to some point of $\mathcal{R}^{N}$,

(ii) $U_{y}$ is in $H_{r}^{2}\left(\mathcal{R}^{N}\right)=\left\{u \in H^{2}\left(\mathcal{R}^{N}\right): u=u(|x|)\right\}$,

(iii) $\lim _{|x| \rightarrow \infty} \mathrm{e}^{|x|}|x|^{\frac{N-1}{2}} u(x)=a>0$,

(iv) the kernel of the linearized operator $L=-\Delta+\left(V(y)-U_{y}^{p-1}\right) \mathrm{Id}$ is spanned by the function $\partial U / \partial x_{i}$ for $i=1, \ldots, N$.

Proof. - See [11] for the uniqueness of the solution and the proof of (ii), [9] for (i) and (iii), [14] for (iv).

It is easily seen that (1.4) is equivalent to

$$
\left\{\begin{array}{l}
-\Delta u+V(\varepsilon x+P) u=u^{p} \quad \text { in } \mathcal{R}^{N}, \\
u>0
\end{array}\right.
$$

for $P \in \mathcal{R}^{N}$.

Now we give the following definitions.

DEFINITION 2.2. - We say that $u_{\varepsilon}$ is a family of bound state solutions of (1.4) if there is a constant $C$ which does not depend on $\varepsilon$ such that

$$
\varepsilon^{N} \int_{\mathcal{R}^{N}}\left(\varepsilon^{2}\left|\nabla u_{\varepsilon}\right|^{2}+u_{\varepsilon}^{2}\right) \mathrm{d} x \leqslant C .
$$

So if we set

$$
v_{\varepsilon}(x)=u_{\varepsilon}(\varepsilon x+P),
$$

where $u_{\varepsilon}$ is a bound state solution of (1.4), we get that $v_{\varepsilon}$ is a solution of (2.3) and

$$
\int_{\mathcal{R}^{N}}\left(\left|\nabla v_{\varepsilon}\right|^{2}+v_{\varepsilon}^{2}\right) \mathrm{d} x \leqslant C .
$$

DEFINITION 2.3. - We say that a family of bound state solution $u_{\varepsilon}$ of (1.4) is singlepeaked if it has only one local maximum point. The point $P_{\varepsilon} \in \mathcal{R}^{N}$ where this maximum is achieved is called the peak of the solution. If $P_{\varepsilon} \rightarrow P$ we say that $u_{\varepsilon}$ concentrates at $P$.

This paper deals with single-peak solution of (1.4). By blow-up arguments ([4]) we get the following 
PROPOSITION 2.4. - Let $u_{\varepsilon}$ be a family of single-peak solution of (1.4) concentrating at $P$ and let $P_{\varepsilon}$ be its peak. If $U_{P}$ is the unique solution of (2.1) symmetric with respect to the origin we have

$$
u_{\varepsilon}(x)=U_{P}\left(\frac{x-P_{\varepsilon}}{\varepsilon}\right)+\omega_{\varepsilon}(x)
$$

and

$$
u_{\varepsilon}(x) \leqslant C \mathrm{e}^{-\left|\frac{x-P_{\varepsilon}}{\varepsilon}\right|},
$$

where $\omega_{\varepsilon} \rightarrow 0$ in $L^{\infty}\left(\mathcal{R}^{N}\right)$.

Proof. - This is a known result (see [4]) but we repeat the proof for reader's convenience. Set $v_{\varepsilon}(x)=u_{\varepsilon}\left(\varepsilon x+P_{\varepsilon}\right)$. Since $u_{\varepsilon}$ is a bound state solution of (1.4), by standard boot-strap arguments we get that $v_{\varepsilon}$ is uniformly bounded on the compact set of $\mathcal{R}^{N}$. Hence, passing to the limit in (2.3) we get that $v_{\varepsilon} \rightarrow U_{P}$ where $U_{P} \in H^{1}\left(\mathcal{R}^{N)}\right.$ solves (1.5) with $y=P$.

Since positive solutions of (1.5) decay exponentially (see [9]) and $U_{P} \in H^{1}\left(\mathcal{R}^{N)}\right.$ is uniformly bounded we obtain the existence of $R>0$ such that $U_{P}(x)<\eta$ for all $|x|=R$. Let us choose $\eta^{p-1}<V_{0}$. But also $u_{\varepsilon}(x)<\eta$ if $|x|=R$ and $n$ large and then, since $v_{\varepsilon}$ has only one local maximum we obtain that $v_{\varepsilon}(x)<\eta$ for all $|x|>R$. Then we can use the maximum principle to conclude that $v_{\varepsilon}(x)<w_{0}(x)$ for all $|x|>R$, where $w_{0}$ is a suitable multiple of the fundamental solution of $\Delta w-b w=0$ with $b=V_{0}-\eta^{p-1}>0$. Since $w_{0}$ decays exponentially we deduce (2.7) and that

$$
v_{\varepsilon} \rightarrow U_{P} \quad \text { uniformly in } \mathcal{R}^{N} .
$$

This ends the proof.

Concerning single-peak solutions Wang (see [18]) proved this useful identity.

PROPOSITION 2.5. - Let us suppose that $u_{\varepsilon}$ is a family of single-peak solutions of (1.4) and

$$
|\nabla V(x)|=\mathrm{O}\left(\mathrm{e}^{\gamma|x|}\right) \quad \text { at infinity for some } \gamma>0
$$

Then

$$
\int_{\mathcal{R}^{N}} \frac{\partial V}{\partial x_{i}}(\varepsilon x+P) u_{\varepsilon}^{2}(\varepsilon x+P) \mathrm{d} x=0 \quad \text { for any } i=1, \ldots, N .
$$

From the previous identity Wang deduced the following result.

THEOREM 2.6. - Let us suppose that V satisfies (2.9) and it is nondecreasing in one direction in $\mathcal{R}^{N}$. Then there is no solution to (1.4).

Proof. - It follows by (2.10).

THEOREM 2.7. - Let $u_{\varepsilon}$ be a family of single-peak solutions to (1.4) and $P_{\varepsilon}$ the point of $\mathcal{R}^{N}$ where $u_{\varepsilon}$ achieves its maximum. Suppose (2.9) holds, $\left\|u_{\varepsilon}-U_{P}\right\|_{H^{1}\left(\mathcal{R}^{N}\right)} \rightarrow 0$ as $\varepsilon \rightarrow 0$. If $V \in C^{1}\left(\mathcal{R}^{N}\right)$ we have that $P_{\varepsilon} \rightarrow P$ with $\nabla V(P)=0$.

Proof. - It follows by (2.10) (see [18]). 


\section{Assumptions on the potential $V$}

This section is devoted to the assumptions on the potential $V$. For $Q \in \mathcal{R}^{N}$ and $\rho \in \mathcal{R}$ let us denote by $B_{Q, \rho}=\left\{x \in \mathcal{R}^{N}\right.$ such that $\left.|x-Q| \leqslant \rho\right\}$. We give the following definition.

DeFINITION 3.1. - We say that a function $h: \mathcal{R}^{N} \mapsto \mathcal{R}$ is homogenous of degree $\alpha \in \mathcal{R}^{+}$with respect to $P \in \mathcal{R}^{N}$ if

$$
h(t x+P)=t^{\alpha} h(x+P) \quad \text { for any } t \in \mathcal{R}^{+} \text {and } x \in \mathcal{R}^{N} .
$$

Remark 3.2. - If $h \in C\left(\mathcal{R}^{N}\right)$ is homogenous of degree $\alpha$ from the previous definition it follows that

$$
|h(x)| \leqslant C|x-P|^{\alpha} \quad \text { for any } x \in \mathcal{R}^{N},
$$

where $C$ is the maximum of $h$ on $\partial B_{P, 1}$.

At this stage we can state our crucial condition on the potential $V$.

Definition of ADMissible POTENTIAL. - Let us assume that $V \in C^{1}\left(R^{N}\right)$ satisfies

$$
|\nabla V(x)| \leqslant C \mathrm{e}^{\gamma|x|} \quad \text { at infinity }
$$

and

$$
0<V_{0} \leqslant V(x) \leqslant V_{1}
$$

for some $\gamma>0$. We say that $V$ is an admissible potential at $P \in R^{N}$ if there exist continuous functions $h_{i}: \mathcal{R}^{N} \mapsto \mathcal{R}, R_{i}: B_{P, r} \mapsto \mathcal{R}$ and real numbers $\alpha_{i} \geqslant 1, i=i, \ldots$, $N$, such that

(i) $\frac{\partial V}{\partial x_{i}}(x)=h_{i}(x)+R_{i}(x)$ in $B_{P, r}$,

(ii) $R_{i}(x) \leqslant C|x-P|^{\beta_{i}}$ in $B_{P, r}$ with $\beta_{1}>\alpha_{i}$ for any $i=1, \ldots, N$,

(iii) $h_{i}(x)=0$ if and only if $x=P$,

(iv) $h_{i}$ is homogeneous of degree $\alpha_{i}$.

Remark 3.3. - We point out that if $P$ an isolated critical point of $V$, (iv) holds and $R_{i}(x) \equiv 0$ then (iii) holds. Indeed if by contradiction there exists $y \in \mathcal{R}^{N}$ such that $h_{i}(y)=0$ then we have that $h_{i}(t(y-P)+P)=0$ for any $t \in \mathcal{R}^{+}$. Hence from (i) of (3.5) we get $\frac{\partial V}{\partial x_{i}}(t(y-P)+P)=0$ for any positive $t$. So $P$ is not an isolated critical point and this gives a contradiction.

Remark 3.4. - It is easy to see that the function $h_{i}$ is determined in an unique way.

Remark 3.5. - The assumptions (i) and (ii) of the previous definition are satisfied if $V \in C^{k}\left(B_{P, r}\right)$ and admits a nonzero derivative with respect to $x_{i}$ for any index $i \in\{1, \ldots, N\}$. In this case $\alpha_{i}+1$ is the order of the first nonzero derivative and the function $h_{i}$ are given by Taylor's formula. In order to state a sufficient condition which implies (iii) we need some notations. Let $\mathbf{k}=\left(k_{1}, k_{2}, \ldots, k_{N}\right)$ be a multi-index and set $|\mathbf{k}|=k_{1}+k_{2}+\cdots+k_{N}, \mathbf{k} !=k_{1} ! k_{2} ! \cdots k_{N} !$, and for $w=\left(w_{1}, w_{2}, \ldots w_{N}\right) \in R^{N}$, 
$w^{\mathbf{k}}=w_{1}^{k_{1}} w_{2}^{k_{2}} \cdots w_{N}^{k_{N}}$. So if

$$
D^{\mathbf{k}} f=\frac{\partial^{|\mathbf{k}|} f}{\partial x_{1}^{k_{1}} \partial x_{2}^{k_{2}} \cdots \partial x_{N}^{k_{N}}}
$$

we have that

$$
h_{i}(x)=\sum_{|\mathbf{k}|=\alpha_{i}+1} \frac{D^{\mathbf{k}} V(P)}{\mathbf{k} !}(x-P)^{\mathbf{k}},
$$

and condition (iii) becomes that the nonlinear system

$$
\sum_{|\mathbf{k}|=\alpha_{i}} \frac{D^{\mathbf{k}} V(P)}{\mathbf{k} !}(x-P)^{\mathbf{k}}=0
$$

admits only the solution $x=P$.

Definition 3.6. - Let $G \in C\left(\mathcal{R}^{N} ; \mathcal{R}^{N}\right)$ be a vector field. We say that y is a stable zero for $G$ if

(i) $G(y)=0$,

(ii) $y$ is isolated,

(iii) if $G_{n}$ is a sequence of vector fields such that $\left\|G_{n}-G\right\|_{C\left(B_{y, \rho}\right)} \rightarrow 0$ for some $\rho>0$ then there exists $y_{n}$ such that $G_{n}\left(y_{n}\right)=0$ and $y_{n} \rightarrow y$.

A sufficient condition on $G$ and $y$ which implies that $y$ is a stable zero is that the index $i(G, y, 0) \neq 0$, where the index of $y$ at zero is given by

$$
i(G, y, 0)=\lim _{\rho \rightarrow 0} \operatorname{deg}\left(G, B_{y, \rho}, 0\right),
$$

with $\operatorname{deg}\left(G, B_{y, \rho}, 0\right)$ denoting the Brouwer degree.

Now let us set

$$
\mathcal{L}_{P}(y)=\left(\int_{R^{N}} h_{i}(x+y+P) U_{P}^{2}(x) \mathrm{d} x\right)_{i=1, \ldots, N},
$$

where $U_{P}$ is the solution of (2.1). By Remark 3.2 and the exponential decay of $U_{P}$ we have that (3.8) is well-posed. Finally set

$$
Z=\left\{y \in \mathcal{R}^{N} \text { such that } y \text { is a stable zero of } \mathcal{L}\right\} .
$$

Remark 3.7. - Condition (1.9) implies that any zero of $\mathcal{L}_{P}$ is stable. Indeed if $\mathcal{L}_{P}(y)=0$ by (1.9) we have that the Brower degree $\operatorname{deg}\left(\mathcal{L}_{P}, B_{P, 1}, 0\right) \neq 0$ (see [13]) and this gives that $y \in Z$.

\section{The existence result}

We start this section by recalling the following result. 
THEOREM 4.1. - Let us assume that $V$ satisfies (3.4). Then there exist positive constant $\delta$ and $\varepsilon_{0}$ such that for every $y$ and $\varepsilon$ with $|y|<\delta$ and $0<\varepsilon<\varepsilon_{0}$ there exists $u_{y, \varepsilon} \in H^{2}\left(\mathcal{R}^{N}\right)$ such that

$$
-\Delta u_{y, \varepsilon}+V(\varepsilon x+y) u_{y, \varepsilon}-\left|u_{y, \varepsilon}\right|^{p-1} u_{y, \varepsilon}=\sum_{i=1}^{N} c_{i, y, \varepsilon} \frac{\partial U_{y}}{\partial x_{i}},
$$

with

$$
\left\|u_{y, \varepsilon}-U_{y}\right\|_{H^{2}\left(\mathcal{R}^{N}\right)} \rightarrow 0 \quad \text { as } \varepsilon \rightarrow 0,
$$

for some constant $c_{i, y, \varepsilon} \in \mathcal{R}$. Here $U_{y}$ is the unique solution of (2.1).

Proof. - The result is classical (see [16] for example).

In the next lemma we give some useful estimates:

LEMMA 4.2. - Let us consider the function $u_{y, \varepsilon}$ given by Theorem 4.1. Then

(i) $u_{y, \varepsilon}$ decays exponentially at the infinity,

(ii) $u_{y, \varepsilon} \rightarrow U_{y}$ uniformly on $\mathcal{R}^{N}$.

Proof. - First of all we show that, for $|y|<\delta, c_{i, y, \varepsilon} \rightarrow 0$ as $\varepsilon \rightarrow 0$ for any $i=$ $\{1, \ldots, N\}$.

Indeed, multiplying (4.1) by $\frac{\partial U_{y}}{\partial x_{j}}$ and integrating we get

$$
\begin{aligned}
c_{j, y, \varepsilon} \int_{\mathcal{R}^{N}}\left(\frac{\partial U}{\partial x_{j}}\right)^{2} & =\sum_{i=0}^{N} c_{i, y, \varepsilon} \int_{\mathcal{R}^{N}} \frac{\partial U}{\partial x_{i}} \frac{\partial U}{\partial x_{j}} \\
& =-\int_{\mathcal{R}^{N}}\left(\Delta u_{y, \varepsilon}+V(\varepsilon x+y) u_{y, \varepsilon}-\left|u_{y, \varepsilon}\right|^{p-1} u_{y, \varepsilon}\right) \frac{\partial U}{\partial x_{j}} \mathrm{~d} x \rightarrow 0 .
\end{aligned}
$$

by (4.2). Then, by using the weak Harnack inequality (see [16], or $[8,18]$ ) it is possible to prove that

$$
u_{y, \varepsilon}(x) \rightarrow 0 \quad \text { uniformly as }|x| \rightarrow \infty .
$$

So there exists $R>0$ such that $V(\varepsilon x+y)-|u|^{p-1} u \geqslant \frac{V_{0}}{2}>0$ for $|x|>R$. At this stage we can repeat the same proof of Lemma 6.4 of [15] which proves (i).

Concerning the proof of (ii) this follows from (i) and the standard boot-strap (here we are using that $1<p<\frac{N+2}{N-2}$ ).

Now we are able to prove the main result of this section.

THEOREM 4.3. - Let us suppose that $V(x)$ is an admissible potential and $\# Z<\infty$. Then there exists $\varepsilon_{0}>0$ such that for any $0<\varepsilon<\varepsilon_{0}$ we have that \#\{single-peak solution of (1.4) concentrating at $P\} \geqslant \# Z$.

Proof. - Let us multiply (4.1) by $\frac{\partial u_{y, \varepsilon}}{\partial x_{j}}$ and integrate. We have that

$$
\int_{\mathcal{R}^{N}}\left(-\Delta u_{y, \varepsilon}-\left|u_{y, \varepsilon}\right|^{p-1} u_{y, \varepsilon}\right) \frac{\partial u_{y, \varepsilon}}{\partial x_{j}} \mathrm{~d} x=0,
$$


(see [18] for a detailed proof) and

$$
\begin{aligned}
\sum_{i=0}^{N} c_{i, y, \varepsilon} \frac{\partial U}{\partial x_{i}} \frac{\partial u_{y, \varepsilon}}{\partial x_{j}} \mathrm{~d} x & =\int_{\mathcal{R}^{N}} V(\varepsilon x+y) u_{y, \varepsilon} \frac{\partial u_{y, \varepsilon}}{\partial x_{j}} \mathrm{~d} x \\
& =-\frac{\varepsilon}{2} \int_{\mathcal{R}^{N}} \frac{\partial V}{\partial x_{j}}(\varepsilon x+y) u_{y, \varepsilon}^{2} \mathrm{~d} x .
\end{aligned}
$$

Let $y_{0} \in Z$ and set $y=P+\varepsilon \tilde{y}$ with $\tilde{y} \in B_{y_{0}, 1}$. By the assumption on $V$ we get

$$
\begin{aligned}
\int_{\mathcal{R}^{N}} \frac{\partial V}{\partial x_{j}}(\varepsilon x+y) u_{y, \varepsilon}^{2} \mathrm{~d} x= & \int_{|\varepsilon x+y-P| \leqslant r}\left(\varepsilon^{\alpha_{i}} h_{j}(x+\tilde{y}+P)+R_{j}(\varepsilon x+y) u_{y, \varepsilon}^{2} \mathrm{~d} x\right) \\
& +\int_{|\varepsilon x+y-P|>r} \frac{\partial V}{\partial x_{j}}(\varepsilon x+y) u_{y, \varepsilon}^{2} \mathrm{~d} x .
\end{aligned}
$$

So by (ii) of (3.5) we deduce

$$
\begin{aligned}
& \quad \int_{|\varepsilon x+y-P| \leqslant r} R_{j}(\varepsilon x+y) \mid u_{y, \varepsilon}^{2} \mathrm{~d} x \\
& \quad \leqslant C \int_{|\varepsilon x+y-P| \leqslant r}|\varepsilon x+y-P|^{\beta_{i}} u_{y, \varepsilon}^{2} \mathrm{~d} x \leqslant C \varepsilon^{\beta_{i}} \int_{\mathcal{R}^{N}}|x+\widetilde{y}|^{\beta} u_{y, \varepsilon}^{2} \mathrm{~d} x \\
& \quad \leqslant C \varepsilon^{\beta_{i}} \int_{\mathcal{R}^{N}}(|x|+1)^{\beta} u_{y, \varepsilon}^{2} \mathrm{~d} x=\mathrm{O}\left(\varepsilon^{\beta_{i}}\right) \quad \text { uniformly with respect to } y .
\end{aligned}
$$

Finally since $\frac{\partial V}{\partial x_{i}}(x)=\mathrm{O}\left(\mathrm{e}^{\gamma|x|}\right)$ at infinity and Lemma 4.2 we have

$$
\begin{aligned}
& \int_{|\varepsilon x+y-P|>r} \frac{\partial V}{\partial x_{i}}(\varepsilon x+y-P) u_{y, \varepsilon}^{2} \mathrm{~d} x \leqslant C \int_{|\varepsilon x+y-P|>r} \mathrm{e}^{\gamma|\varepsilon x+y|} \mathrm{e}^{-2|x|} \mathrm{d} x \\
& \leqslant C \int_{|x|>\frac{r}{2 \varepsilon}} \mathrm{e}^{-|x|}=\mathrm{O}\left(\mathrm{e}^{-\frac{r}{\varepsilon}}\right) \text { uniformly with respect to } y .
\end{aligned}
$$

Then by (4.7), (4.8) and Lemma 4.2 we get

$$
\sum_{i=0}^{N} c_{i, y, \varepsilon} \int_{\mathcal{R}^{N}} \frac{\partial U}{\partial x_{i}} \frac{\partial u_{y, \varepsilon}}{\partial x_{j}} \mathrm{~d} x=\varepsilon^{\alpha_{i}}\left(\int_{\mathcal{R}^{N}} h_{j}(x+y+P) u_{y, \varepsilon}^{2} \mathrm{~d} x+\mathrm{O}\left(\varepsilon^{\beta_{i}-\alpha_{i}}\right)\right) .
$$

Again by (ii) of Lemma 4.2 and Remark 3.2 we obtain that

$$
\int_{|\varepsilon x+y-P| \leqslant r} h_{j}(x+\tilde{y}+P) u_{y, \varepsilon}^{2} \mathrm{~d} x \rightarrow \int_{\mathcal{R}^{N}} h_{j}(x+\tilde{y}+P) U_{y}^{2} \mathrm{~d} x
$$


uniformly in $B_{y_{0}, 1}$. So by the definition of stable zero we get that there exists $y_{\varepsilon} \rightarrow y_{0}$ such that

$$
\int_{\mathcal{R}^{N}} \frac{\partial V}{\partial x_{j}}\left(\varepsilon x+y_{\varepsilon}\right) u_{y_{\varepsilon}, \varepsilon}^{2} \mathrm{~d} x=0 .
$$

Thus

$$
\sum_{i=0}^{N} c_{i, y_{\varepsilon}, \varepsilon} \frac{\partial U_{P+\varepsilon y_{\varepsilon}}}{\partial x_{i}} \frac{\partial u_{P+\varepsilon y_{\varepsilon}}}{\partial x_{j}} \mathrm{~d} x=0 .
$$

From the uniqueness of the solution of (2.1) and the implicit function theorem we get that $U_{P+\varepsilon y_{\varepsilon}} \rightarrow U_{P}$ in $H^{2}\left(\mathcal{R}^{N}\right)$ (see Lemma 4.1 of [8] for a detailed proof). Then

$$
\int_{\mathcal{R}^{N}} \frac{\partial U_{P+\varepsilon y_{\varepsilon}}}{\partial x_{i}} \frac{\partial u_{P+\varepsilon y_{\varepsilon}}}{\partial x_{j}} \mathrm{~d} x \rightarrow \delta_{i}^{j} \int_{\mathcal{R}^{N}}\left(\frac{\partial U_{P}}{\partial x_{i}}\right)^{2} \neq 0
$$

and this implies that, for $\varepsilon$ small, the linear system (4.13) admits only the trivial solution $c_{i, y_{\varepsilon}, \varepsilon}=0$ for any $i=\{1, \ldots, N\}$. This proves that, for any stable zero $y_{0} \in Z$ there exists a solution $u_{\varepsilon}$ of (2.3) such that

$$
\left\|u_{\varepsilon}-U_{P}\right\|_{H^{2}\left(\mathcal{R}^{N}\right) \cap L^{\infty}\left(\mathcal{R}^{N}\right)} \rightarrow 0 \quad \text { as } \varepsilon \rightarrow 0 .
$$

By standard arguments (see [8] or [18] for example), we get that $u_{\varepsilon}>0$ in $\mathcal{R}^{N}$. In order to prove our claim we have to show that two different stable zeroes $y_{1}$ and $y_{2}$ generates two different solutions.

Set $u_{1, \varepsilon}$ and $u_{2, \varepsilon}$ the solutions of (2.3) generates by $y_{1}$ and $y_{2}$ and $y_{\varepsilon}^{1} \rightarrow y_{1}, y_{\varepsilon}^{2} \rightarrow y_{2}$ with $y_{1} \neq y_{2}$. We recall that $u_{1, \varepsilon}$ and $u_{2, \varepsilon}$ solve

$$
-\Delta u_{1, \varepsilon}+V\left(\varepsilon x+P+\varepsilon y_{\varepsilon}^{1}\right) u_{1, \varepsilon}=u_{1, \varepsilon}^{p},
$$

and

$$
-\Delta u_{2, \varepsilon}+V\left(\varepsilon x+P+\varepsilon y_{\varepsilon}^{2}\right) u_{2, \varepsilon}=u_{2, \varepsilon}^{p} .
$$

Let

$$
z_{1, \varepsilon}(x)=u_{1, \varepsilon}\left(\frac{x-\left(P+\varepsilon y_{\varepsilon}^{1}\right)}{\varepsilon}\right)
$$

and

$$
z_{2, \varepsilon}(x)=u_{2, \varepsilon}\left(\frac{x-\left(P+\varepsilon y_{\varepsilon}^{2}\right)}{\varepsilon}\right)
$$

the corresponding solutions of (1.4). By (4.15) we deduce that

$$
\begin{aligned}
& z_{1, \varepsilon}\left(P+\varepsilon y_{\varepsilon}^{1}\right)=u_{1, \varepsilon}(0) \rightarrow U_{P}(0), \\
& z_{2, \varepsilon}\left(P+\varepsilon y_{\varepsilon}^{1}\right)=u_{1, \varepsilon}\left(y_{\varepsilon}^{1}-y_{\varepsilon}^{2}\right) \rightarrow U_{P}\left(y_{1}-y_{2}\right) \neq U_{P}(0) .
\end{aligned}
$$

This proves that $u_{1, \varepsilon} \neq u_{2, \varepsilon}$. 


\section{A crucial estimate}

The aim of this section is to compute asymptotics for the ratio

$$
\frac{P-P_{\varepsilon}}{\varepsilon},
$$

where $P_{\varepsilon}$ is the peak of $u_{\varepsilon}$. We know that $P_{\varepsilon} \rightarrow P$ as $\varepsilon \rightarrow 0$. Next proposition gives more information.

Proposition 5.1. - Assume that $V(x)$ is an admissible potential and $u_{\varepsilon}$ is a solution of (1.4). Then there exists a positive constant $C$ such that

$$
\left|\frac{P-P_{\varepsilon}}{\varepsilon}\right| \leqslant C .
$$

Proof. - By contradiction let us suppose that there exists a sequence $\varepsilon_{n} \rightarrow 0$ such that

$$
\frac{\left|P_{\varepsilon_{n}}-P\right|}{\varepsilon_{n}} \rightarrow \infty
$$

Set $P_{n}=P_{\varepsilon_{n}}$ and $v_{n}(x)=u_{\varepsilon_{n}}\left(\varepsilon_{n} x+P_{n}\right)$. By Proposition $2.4 v_{n} \rightarrow U_{P}$ in $L^{2}\left(\mathcal{R}^{N}\right)$ and then

$$
\int_{R^{N}} \frac{\partial V}{\partial x_{i}}\left(\varepsilon_{n} x+P_{n}\right) v_{n}^{2}(x) \mathrm{d} x=0 .
$$

Hence

$$
\begin{aligned}
\int_{R^{N}} & \frac{\partial V}{\partial x_{i}}\left(\varepsilon_{n} x+P_{n}\right) v_{n}^{2}(x) \mathrm{d} x \\
= & \int_{\left|\varepsilon_{n} x+P_{n}-P\right| \leqslant r} h_{i}\left(\varepsilon_{n} x+P_{n}\right) v_{n}^{2}(x) \mathrm{d} x+\int_{\left|\varepsilon_{n} x+P_{n}-P\right| \leqslant r} R_{i}\left(\varepsilon_{n} x+P_{n}\right) v_{n}^{2}(x) \mathrm{d} x \\
& +\int_{\left|\varepsilon_{n} x+P_{n}-P\right|>r} \frac{\partial V}{\partial x_{i}}\left(\varepsilon_{n} x+P_{n}\right) v_{n}^{2}(x) \mathrm{d} x .
\end{aligned}
$$

Concerning the first integral we have

$$
\begin{aligned}
& \int_{\left|\varepsilon_{n} x+P_{n}-P\right| \leqslant r} h_{i}\left(\varepsilon_{n} x+P_{n}\right) v_{n}^{2}(x) \mathrm{d} x \\
= & \left|P_{n}-P\right|^{\alpha_{i}} \int_{\left|\varepsilon_{n} x+P_{n}-P\right| \leqslant r} h_{i}\left(\frac{\varepsilon_{n} x}{\left|P_{n}-P\right|}+\frac{P_{n}-P}{\left|P_{n}-P\right|}+P\right) v_{n}^{2}(x) \mathrm{d} x .
\end{aligned}
$$

Up to a subsequence $\frac{P_{n}-P}{\left|P_{n}-P\right|} \rightarrow \zeta$ with $|\zeta|=1$. Then we get

$$
I_{1, n}=\left|P_{n}-P\right|^{\alpha_{i}} h_{i}(\zeta+P) \int_{\mathcal{R}^{N}} U^{2}(x) \mathrm{d} x+\mathrm{o}\left(\left|P_{n}-P\right|^{\alpha_{i}}\right) .
$$


Moreover, from the definition of $R_{i}$,

$$
\begin{aligned}
\left|I_{2, n}\right| & \leqslant C \int_{\left|\varepsilon_{n} x+P_{n}-P\right| \leqslant r}\left|\varepsilon_{n} x+P_{n}-P\right|^{\beta_{i}} v_{n}^{2}(x) \mathrm{d} x \\
& =C\left|P_{n}-P\right|^{\beta_{i}} \int_{\mathcal{R}^{N}} U^{2}(x) \mathrm{d} x+\mathrm{o}\left(\left|P_{n}-P\right|^{\beta_{i}}\right) .
\end{aligned}
$$

Finally since $\frac{\partial V}{\partial x_{i}}(x)=\mathrm{O}\left(\mathrm{e}^{\gamma x}\right)$ at infinity and (iii) of Theorem 2.1 we have

$$
\begin{aligned}
I_{3, n} & =\int_{\left|\varepsilon_{n} x+P_{n}-P\right|>r} \frac{\partial V}{\partial x_{i}}\left(\varepsilon_{n} x+P_{n}\right) v_{n}^{2}(x) \mathrm{d} x \leqslant C \int_{\left|\varepsilon_{n} x+P_{n}-P\right|>r} \mathrm{e}^{\gamma\left|\varepsilon_{n} x+P_{n}\right|} \mathrm{e}^{-2|x|} \mathrm{d} x \\
& \leqslant \int_{|x|>r / 2 \varepsilon_{n}} \mathrm{e}^{-|x|}=\mathrm{O}\left(\mathrm{e}^{-\frac{r}{\varepsilon_{n}}}\right) .
\end{aligned}
$$

Thus (5.5) becomes

$$
h_{i}(\zeta+P) \int_{\mathcal{R}^{2}} U^{2}(x) \mathrm{d} x=\mathrm{o}\left(\left|P_{n}-P\right|^{\beta_{i}-\alpha_{i}}\right)+\frac{\mathrm{e}^{-\frac{\gamma}{\varepsilon_{n}}}}{\left|P_{n}-P\right|^{\alpha_{i}}} .
$$

This clearly implies $h_{i}(\zeta+P)=0$ and since $|\zeta|=1$ we have a contradiction with (iii) of (3.5).

PROPOSITION 5.2. - Let $\varepsilon_{n}$ be a sequence which goes to zero and $u_{\varepsilon_{n}}=u_{n}$ the corresponding solutions of (1.4). Then, up to a subsequence, if $P_{n}$ denotes the peak of the solution $u_{n}$ we have

$$
\frac{P_{n}-P}{\varepsilon_{n}} \rightarrow y
$$

where y satisfies $\mathcal{L}(y)=0$.

Proof. - By the previous proposition we have that $\frac{P-P_{n}}{\varepsilon_{n}}$ is bounded and so we can assume that (up to a subsequence) $\frac{P-P_{n}}{\varepsilon_{n}} \rightarrow y$.

Again as in (5.5) we get

$$
0=\int_{R^{N}} \frac{\partial V}{\partial x_{i}}\left(\varepsilon_{n} x+P_{n}\right) v_{\varepsilon_{n}}^{2}(x) \mathrm{d} x=I_{1, n}+I_{2, n}+I_{3, n} .
$$

Now

$$
\begin{aligned}
I_{1, n} & =\varepsilon_{n}^{\alpha_{i}} \int_{\left|\varepsilon_{n} x+P_{n}-P\right| \leqslant r} h_{i}\left(x+\frac{P_{n}-P}{\varepsilon_{n}}+P\right) v_{\varepsilon_{n}}^{2}(x) \mathrm{d} x \\
& =\varepsilon_{n}^{\alpha_{i}} \int_{\mathcal{R}^{N}} h_{i}(x+y+P) U^{2}(x) \mathrm{d} x+\mathrm{o}(1) .
\end{aligned}
$$

Proceeding as in Proposition 5.2 we get

$$
I_{2, n}=\mathrm{O}\left(\varepsilon_{n}^{\beta_{i}}\right),
$$


and

$$
I_{3, n}=\mathrm{O}\left(\mathrm{e}^{-\gamma / \varepsilon_{n}}\right)
$$

Then (5.12) becomes

$$
\int_{\mathcal{R}^{N}} h_{i}(x+y+P) U^{2}(x) \mathrm{d} x=\mathrm{O}\left(\varepsilon_{n}^{\beta_{i}-\alpha_{i}}\right) .
$$

Passing to the limit in (5.16) we get the claim.

From the previous proposition we get the following "local" nonexistence result

COROLLARY 5.3. - Let us suppose that $V$ is an admissible potential and $\mathcal{L}(y) \neq 0$ for any $y$. Then there is no single peak solution of (1.4) concentrating at $P$.

In the next lemmas we show some cases where $Z$ is nonempty.

Lemma 5.4. - Let $P$ be a nondegenerate critical point of $V \in C^{3}\left(B_{P, r}\right)$. Then $Z=\{0\}$.

Proof. - By Taylor's formula we have that

$$
V(x)=V(P)+\frac{1}{2} \sum_{j, k=1}^{N} \frac{\partial^{2} V}{\partial x_{j} \partial x_{k}}(P)\left(x_{j}-P_{j}\right)\left(x_{k}-P_{k}\right)+R(x) .
$$

So in this case

$$
h_{i}(x)=\sum_{j=1}^{N} \frac{\partial^{2} V}{\partial x_{i} \partial x_{j}}(P)\left(x_{j}-P_{j}\right) \quad \text { and } \quad R_{i}(x)=\frac{\partial R(x)}{\partial x_{i}} .
$$

Then

$$
0=\sum_{j=1}^{N} \frac{\partial^{2} V}{\partial x_{i} \partial x_{j}}(P) \int_{R^{N}}\left(x_{i}+y_{i}\right) U^{2}(x) \mathrm{d} x=\sum_{i=1}^{N} \frac{\partial^{2} V}{\partial x_{i} \partial x_{j}}(P) \int_{R^{N}} y_{i} U^{2}(x) \mathrm{d} x .
$$

Since the matrix $\frac{\partial^{2} V}{\partial x_{i} \partial x_{j}}(P)$ is invertible we have that the linear system

$$
\sum_{i=1}^{N} \frac{\partial^{2} V}{\partial x_{i} \partial x_{j}}(P) y_{i}=0
$$

admits only the solution $y=0$. Moreover, again by the invertibility of the matrix $\frac{\partial^{2} V}{\partial x_{i} \partial x_{j}}(P)$ we have $\operatorname{det} \operatorname{Jac} \mathcal{L}_{P}(0) \neq 0$ and so $y$ is a zero stable of $h_{i}$.

More in general we have the following

LEMMA 5.5. - Let us suppose that $V(x)=c_{0}+\sum_{i=1}^{m} a_{i} x_{i}^{\alpha_{i}+1}$ in a neighborhood of $P=0$. Here $\alpha_{i}$ are positive even integers, $a_{i} \in R$ with $a_{i} \neq 0$ and $c_{0}>0$. Then $Z=\{0\}$. 
Proof. - We have that

$$
h_{i}(x)=\left(\alpha_{i}+1\right) a_{i} \int_{R^{N}} x_{i}^{\alpha_{i}} U^{2}(x) \mathrm{d} x .
$$

Let us look for the zeroes of $h_{i}$.

$$
\int_{R^{N}}\left(x_{i}+y_{i}\right)^{\alpha_{i}} U^{2}(x) \mathrm{d} x=0 \quad \text { for any } i=1, \ldots, N .
$$

Since $\alpha_{i}$ are even numbers and $U$ is an even function we get

$$
\begin{aligned}
\int_{R^{N}}\left(x_{i}+y_{i}\right)^{\alpha_{i}} U^{2}(x) \mathrm{d} x & =\sum_{k=0}^{\alpha_{i}}\left(\begin{array}{c}
\alpha_{i} \\
k
\end{array}\right) \int_{R^{N}} x_{i}^{k} y_{i}^{\alpha_{i}-k} U^{2}(x) \mathrm{d} x \\
& =\sum_{k=0}^{\frac{\alpha_{i}}{2}}\left(\begin{array}{c}
\alpha_{i} / 2 \\
2 k
\end{array}\right) \int_{R^{N}} x_{i}^{2 k} y_{i}^{\alpha_{i}-2 k} U^{2}(x) \mathrm{d} x .
\end{aligned}
$$

Because of the function $\sum \gamma_{i} z^{\delta_{i}}$ is strictly increasing if $\gamma_{i}$ is positive and $\delta_{i}$ is odd we get that (5.22) admit only the solution $y=0$. Moreover it is easy to verify that $\operatorname{det} \operatorname{Jac} \mathcal{L}_{P}(0) \neq 0$ and so $Z=\{0\}$.

As application of Corollary 5.3 we obtain the following nonexistence result.

PROPOSITION 5.6. - Let us suppose that $V(x)=c_{0}+\sum_{j=1}^{m} a_{j} x^{\alpha_{j}}$ in a neighborhood of $x=0$. Assume that at least one of the integers $\alpha_{j}$ is odd, $a_{j} \in R$ with $a_{j} \neq 0$ and $c_{0}>0$. Then there is no solution $u_{\varepsilon}$ concentrating at $P=0$.

\section{An exact multiplicity result}

In this section we prove Theorem 1.1. Let us start with the following

Proposition 6.1. - Let $\varepsilon_{n}$ be a sequence which goes to zero and $u_{n}$ the corresponding single-peak solution of (1.4). Let $P_{n}$ the peaks of $u_{n}$ and assume that (5.11) holds. Then

$$
u_{n}(x)-U_{P}\left(\frac{x-P}{\varepsilon_{n}}-y\right) \rightarrow 0 \quad \text { uniformly in } \mathcal{R}^{N} .
$$

Proof. - Let us consider $v_{n}(x)=u_{n}\left(\varepsilon_{n} x+P_{n}\right)$. So $v_{n}$ is a positive solutions of

$$
\Delta v_{n}+V\left(\varepsilon_{n} x+P\right) v_{n}=v_{n}^{p} \quad \text { in } \mathcal{R}^{N} .
$$

Proceeding as in the proof of Proposition 2.7 we get

$$
v_{n} \rightarrow U_{P} \quad \text { uniformly in } \mathcal{R}^{N} .
$$


Moreover $\left\|v_{n}\right\|_{\infty} \geqslant V_{0}^{1 /(p-1)}$ and then we get that $U_{P} \not \equiv 0$. Since

$$
v_{n}\left(\frac{P_{n}-P}{\varepsilon_{n}}\right)=u_{n}\left(P_{n}\right)=\max _{x \in \mathcal{R}^{N}} u_{n}(x)
$$

we derive

$$
U_{P}(y)=\max _{x \in \mathcal{R}^{N}} U_{P}(x) .
$$

But $U_{P}$ is radially symmetric with respect to some point (see [9]) so that $U_{P}(x)=$ $U_{P}(|x-y|)$. This proves the claim.

Now we are able to prove Theorem 1.1.

Proof of Theorem 1.1. - By Theorem 4.3 we know that \#\{single-peak solution of (1.4) concentrating at $x=P\} \geqslant \# Z$. By contradiction let us suppose that \#\{single-peak solution of (1.4) concentrating at $x=P\}>\# Z$. Since $\# Z<\infty$ and Proposition 5.2 there exist $y \in Z, \varepsilon_{n} \rightarrow 0$ and two distinct positive solutions $u_{1, n}$ and $u_{2, n}$ of (1.4) with $\varepsilon=\varepsilon_{n}$ such that if $P_{1, n}$ and $P_{2, n}$ are their peaks we have

$$
\lim _{n \rightarrow \infty} \frac{P_{1, n}-P}{\varepsilon_{n}}=\lim _{n \rightarrow \infty} \frac{P_{2, n}-P}{\varepsilon_{n}}=y .
$$

From Proposition 6.1 the functions $v_{1, n}(x)=u_{1, n}\left(\varepsilon_{n} x+P\right)$ and $v_{2, n}(x)=u_{2, n}\left(\varepsilon_{n} x+\right.$ $P$ ) satisfy $v_{1, n}(x), v_{2, n}(x) \rightarrow U_{P}(|x-y|)$ uniformly on $\mathcal{R}^{N}$.

Since $u_{1, n} \not \equiv u_{2, n}$ we can consider

$$
w_{n}(x)=\frac{v_{1, n}-v_{2, n}}{\left\|v_{1, n}-v_{2, n}\right\|_{\infty}} \quad \text { in } \mathcal{R}^{N},
$$

and so $w_{n}$ satisfies

$$
-\Delta w_{n}+V\left(\varepsilon_{n} x+P\right) w_{n}=c_{n}(x) w_{n} \quad \text { in } \mathcal{R}^{N}
$$

with

$$
c_{n}(x)=p \int_{0}^{1}\left(t v_{1, n}(x)+(1-t) v_{2, n}(x)\right)^{p-1} \mathrm{~d} t,
$$

and $c_{n}(x) \rightarrow U_{P}^{p-1}(|x-y|)$ in $C_{\mathrm{loc}}^{1}\left(\mathcal{R}^{N}\right) \cap L^{\infty}\left(\mathcal{R}^{N}\right)$.

Passing to the limit in (6.6) we get that $w_{n} \rightarrow w$ in $C_{\text {loc }}^{1}\left(\mathcal{R}^{N}\right)$ where $w$ solves

$$
-\Delta w+V(P) w=p U_{P}(|x-y|)^{p-1} w \quad \text { in } \mathcal{R}^{N} .
$$

By (iv) of Theorem 2.1 we obtain that

$$
w(x)=\sum_{j=1}^{N} a_{j} \frac{\partial U_{P}(|x-y|)}{\partial x_{j}}
$$


with $a_{j} \in \mathcal{R}$.

Now the proof will be divided in two steps.

Step 1: we have that $a_{j}=0$ for any $j=1, \ldots, N$.

From (2.10) we get

$$
\int_{\mathcal{R}^{N}} \frac{\partial V}{\partial x_{i}}\left(\varepsilon_{n} x+P\right) v_{1, n}^{2}(x) \mathrm{d} x=\int_{\mathcal{R}^{N}} \frac{\partial V}{\partial x_{i}}\left(\varepsilon_{n} x+P\right) v_{2, n}^{2}(x) \mathrm{d} x=0 .
$$

Then we have

$$
\int_{\mathcal{R}^{N}} \frac{\partial V}{\partial x_{i}}\left(\varepsilon_{n} x+P\right) w_{n}(x)\left(v_{1, n}(x)+v_{2, n}(x)\right) \mathrm{d} x=0 .
$$

Since $\left|w_{n}\right| \leqslant 1$ and $v_{1, n}$ and $v_{2, n}$ decay exponentially (see Lemma 4.2) we can proceed as in the previous sections. So by (6.10) we obtain

$$
\sum_{j=1}^{N} a_{j} \int_{\mathcal{R}^{N}} h_{j}(x+P) \frac{\partial U_{P}(|x-y|)}{\partial x_{i}} U_{P}(|x-y|) \mathrm{d} x=0 .
$$

Recalling the definition of $\mathcal{L}_{P}$ we have

$$
\operatorname{Jac} \mathcal{L}_{P}(y)=\left(2 \int_{\mathcal{R}^{N}} h_{j}(x+P) \frac{\partial U_{P}(|x-y|)}{\partial x_{i}} U_{P}(|x-y|) \mathrm{d} x\right)_{i, j=1, \ldots, N} .
$$

Hence (1.9) implies that the linear system (6.11) admits only the trivial solution $a_{j}=0$ for any $j=1, \ldots, N$. So $w \equiv 0$.

Step 2: $w \equiv 0$ cannot occur.

Let $x_{n}$ be such that $w_{n}\left(x_{n}\right)=\left\|w_{n}\right\|_{\infty}=1$ (the same proof applies if $w_{n}\left(x_{n}\right)=-1$ ). If $\left|x_{n}\right| \leqslant C$ a contradiction arises since by the previous step $w_{n} \rightarrow 0$ in $C_{\mathrm{loc}}^{1}\left(R^{N}\right)$. On the other hand if $\left|x_{n}\right| \rightarrow \infty$ we have $\Delta w_{n}\left(x_{n}\right) \leqslant 0$ and by (6.6) $V_{0} \leqslant V\left(\varepsilon_{n} x+P\right)=c_{n}\left(x_{n}\right)$. This gives a contradiction since $c_{n}\left(x_{n}\right) \rightarrow 0$ and so the claim of the theorem follows.

THEOREM 6.2. - Let $V$ be an admissible potential and suppose that (1.9) holds. Then for $\varepsilon$ small enough any single-peak solution $u_{\varepsilon}$ of (1.4) is nondegenerate, i.e., the linear problem

$$
\left\{\begin{array}{l}
-\varepsilon^{2} \Delta \phi+V(x) \phi=u_{\varepsilon}^{p-1} \phi \quad \text { in } \mathcal{R}^{N}, \\
v \in H^{1}\left(R^{N}\right) .
\end{array}\right.
$$

admits only the trivial solution $\phi \equiv 0$.

Proof. - By contradiction let us suppose that there exists a sequence $\varepsilon_{n} \rightarrow 0$ and a solution $\phi_{n}$ of (6.13) with $u_{\varepsilon}=u_{\varepsilon_{n}}$ such that

$$
\left\{\begin{array}{l}
-\varepsilon_{n}^{2} \Delta \phi_{n}+V(x) \phi_{n}=u_{\varepsilon_{n}}^{p-1} \phi_{n} \quad \text { in } \mathcal{R}^{N}, \\
\phi \in H^{1}\left(R^{N}\right) .
\end{array}\right.
$$


Set $u_{\varepsilon_{n}}=u_{n}$ and let us denote by $P_{n}$ the peak of the solution $u_{n}$. Consider $\widetilde{\phi}_{n}(x)=$ $\phi_{n}\left(\varepsilon_{n} x+P\right)$. Since $V$ is admissible $\frac{P_{n}-P}{\varepsilon_{n}} \rightarrow y$ with $y \in Z$ and $\widetilde{\phi}_{n}(x)$ satisfies

$$
\left\{\begin{array}{l}
-\Delta \widetilde{\phi}_{n}+V\left(\varepsilon_{n} x+P_{n}\right) \widetilde{\phi}_{n}=u_{n}^{p-1}\left(\varepsilon_{n} x+P_{n}\right) \widetilde{\phi}_{n} \quad \text { in } \mathcal{R}^{N}, \\
\widetilde{\phi}_{n} \in H^{1}\left(R^{N}\right) .
\end{array}\right.
$$

Finally set

$$
v_{n}=\frac{\widetilde{\phi}_{n}}{\left\|\widetilde{\phi}_{n}\right\|_{L^{\infty}\left(\mathcal{R}^{N}\right)}} .
$$

As in the previous theorem it is easily seen that

$$
v_{n}(x) \rightarrow \sum_{j=1}^{N} a_{j} \frac{\partial U_{P}(|x-y|)}{\partial x_{j}} \quad \text { in } C_{\mathrm{loc}}^{1}\left(\mathcal{R}^{N}\right) .
$$

Let us write down the equation satisfied by $\frac{\partial v_{n}}{\partial x_{i}}$

$$
\left\{\begin{array}{l}
-\Delta \frac{\partial v_{n}}{\partial x_{i}}+V\left(\varepsilon_{n} x+P\right) \frac{\partial v_{n}}{\partial x_{i}}+\varepsilon_{n} \frac{V\left(\varepsilon_{n} x+P\right)}{\partial x_{i}} v_{n}=u_{n}^{p-1} \frac{\partial v_{n}}{\partial x_{i}} \quad \text { in } \mathcal{R}^{N}, \\
v \in H^{1}\left(R^{N}\right) .
\end{array}\right.
$$

Multiplying (6.14) by $\frac{\partial u_{n}}{\partial x_{i}}$ and (6.15) by $v_{n}$ we get

$$
\int_{\mathcal{R}^{N}} \frac{\partial V\left(\varepsilon_{n} x+P\right)}{\partial x_{i}} \frac{\partial u_{n}}{\partial x_{i}} v_{n} \mathrm{~d} x=0
$$

Passing to the limit in (6.15) we have

$$
\sum_{j=1}^{N} a_{j} \int_{\mathcal{R}^{N}} h_{i}(x+P) U_{P}(|x-y|) \frac{\partial U_{P}(|x-y|)}{\partial x_{j}} \mathrm{~d} x=0 .
$$

As in the previous theorem, by (1.9) we obtain $a_{j}=0$ for any $j=1, \ldots, N$. So $v_{n} \rightarrow 0$ in $C_{\text {loc }}^{1}\left(\mathcal{R}^{N}\right)$ and from this a contradiction follows (see Step 2 of the previous theorem).

Example. - Here we show an example of a potential $V$ such that (1.4) admits exactly two single-peak solutions concentrating at $P$.

Let $V\left(x_{1}, x_{2}\right) \in C^{1}\left(\mathcal{R}^{N}\right) \cap L^{\infty}\left(\mathcal{R}^{N}\right)$ such that $V\left(x_{1}, x_{2}\right)=V_{0}+x_{1}^{3}-x_{1} x_{2}^{2}$ in $B_{0,1}$, $V_{0}>0$. Of course 0 is an isolated critical point of $V$ and $V$ is admissible.

By direct computation we have that, for $y=\left(y_{1}, y_{2}\right)$,

$$
\begin{aligned}
\mathcal{L}_{0}(y)= & \left(2 \int_{\mathcal{R}^{N}} x_{1}^{2} U^{2}\left(x_{1}, x_{2}\right) \mathrm{d} x_{1} \mathrm{~d} x_{2}+\left(3 y_{1}^{2}-y_{2}^{2}\right) \int_{\mathcal{R}^{N}} U^{2}\left(x_{1}, x_{2}\right) \mathrm{d} x_{1} \mathrm{~d} x_{2},\right. \\
& \left.-6 y_{1} y_{2} \int_{\mathcal{R}^{N}} U^{2}\left(x_{1}, x_{2}\right) \mathrm{d} x_{1} \mathrm{~d} x_{2}\right) .
\end{aligned}
$$


Moreover

$$
\mathcal{L}\left(z_{1}\right)=\mathcal{L}\left(z_{2}\right)=0
$$

for

$z_{1}=\left(0,2 \frac{\int_{\mathcal{R}^{N}} x_{1}^{2} U^{2}\left(x_{1}, x_{2}\right) \mathrm{d} x_{1} \mathrm{~d} x_{2}}{\int_{\mathcal{R}^{N}} U^{2}\left(x_{1}, x_{2}\right) \mathrm{d} x_{1} \mathrm{~d} x_{2}}\right) \quad$ and $\quad z_{2}=\left(0,-2 \frac{\int_{\mathcal{R}^{N}} x_{1}^{2} U^{2}\left(x_{1}, x_{2}\right) \mathrm{d} x_{1} \mathrm{~d} x_{2}}{\int_{\mathcal{R}^{N}} U^{2}\left(x_{1}, x_{2}\right) \mathrm{d} x_{1} \mathrm{~d} x_{2}}\right)$.

If we prove that $\operatorname{Jac} \mathcal{L}\left(z_{1}\right)$ and $\operatorname{Jac} \mathcal{L}\left(z_{2}\right)$ are invertible matrixes by Theorem 1.1 and Remark 3.7 we deduce the claim. Indeed

$$
\operatorname{Jac} \mathcal{L}_{0}(y)=\left(\begin{array}{cc}
6 y_{1} \int_{\mathcal{R}^{N}} U^{2}\left(x_{1}, x_{2}\right) \mathrm{d} x_{1} \mathrm{~d} x_{2} & -2 y_{2} \int_{\mathcal{R}^{N}} U^{2}\left(x_{1}, x_{2}\right) \mathrm{d} x_{1} \mathrm{~d} x_{2} \\
-6 y_{2} \int_{\mathcal{R}^{N}} U^{2}\left(x_{1}, x_{2}\right) \mathrm{d} x_{1} \mathrm{~d} x_{2} & -6 y_{1} \int_{\mathcal{R}^{N}} U^{2}\left(x_{1}, x_{2}\right) \mathrm{d} x_{1} \mathrm{~d} x_{2}
\end{array}\right)
$$

and $\operatorname{det} \operatorname{Jac} \mathcal{L}\left(z_{1}\right)=\operatorname{det} \operatorname{Jac} \mathcal{L}\left(z_{2}\right) \neq 0$.

We point out that with a little bit of computation it is possible to construct an example of $V$ such that (1.4) admits $k$ solution which "concentrate" in the same point $P$. We give a short proof of this.

Set $V\left(x_{1}, x_{2}\right) \in C^{1}\left(\mathcal{R}^{N}\right) \cap L^{\infty}\left(\mathcal{R}^{N}\right)$ such that

$$
V\left(x_{1}, x_{2}\right)=V_{0}+\sum_{i, j=1}^{L} a_{i j} x_{1}^{i} x_{2}^{j} \quad \text { with } a_{i j} \in \mathcal{R} \text { and } L \in N .
$$

We choose $a_{i j}$ so that the following system

$$
\left\{\begin{array}{l}
\sum_{i, j=1}^{L} \sum_{m=0}^{i-1} \sum_{h=0}^{j} i a_{i j}\left(\begin{array}{c}
h \\
j
\end{array}\right)\left(\begin{array}{c}
m \\
i-1
\end{array}\right) y_{1}^{i-1-m} y_{2}^{j-h} \int_{\mathcal{R}^{N}} x_{1}^{m} x_{2}^{h} U^{2}\left(x_{1}, x_{2}\right) \mathrm{d} x_{1} \mathrm{~d} x_{2}=0, \\
\sum_{i, j=1}^{L} \sum_{m=0}^{i} \sum_{h=0}^{j-1} j a_{i j}\left(\begin{array}{c}
h \\
j-1
\end{array}\right)\left(\begin{array}{c}
m \\
i
\end{array}\right) y_{1}^{i-m} y_{2}^{j-h-1} \int_{\mathcal{R}^{N}} x_{1}^{m} x_{2}^{h} U^{2}\left(x_{1}, x_{2}\right) \mathrm{d} x_{1} \mathrm{~d} x_{2}=0
\end{array}\right.
$$

admits $k$ solutions $z_{1}, z_{2}, \ldots, z_{k} \in \mathcal{R}^{2}$. It is also possible to prove that we can choose $a_{i j}$ in order to have that

$$
\operatorname{det} \operatorname{Jac} \mathcal{L}\left(z_{i}\right) \neq 0 \quad \text { for any } i=1, \ldots, k .
$$

and this proves the claim.

The shape of $V$ near its critical point $P$ plays an important role for multiplicity results of (2.3) of solutions concentrating at a point $P$. Next proposition tries to give partial answers in this direction.

PROPOSITION 6.3. - Let us suppose that

$$
V(x)=c_{0}+\sum_{i=1}^{m} a_{i} x_{i}^{\alpha_{i}+1}
$$


in a neighborhood of $x=0$. Here $\alpha_{i}$ are positive even integers, $a_{i} \in R$ with $a_{i} \neq 0$ and $c_{0}>0$. Then either

(i) at least one of the integers $\alpha_{i}$ is even and then there is no solution of (2.3) or

(ii) the integers $\alpha_{i}$ are odd and then there is only one nondegenerate solution of (2.3) which concentrates at $x=P$.

Proof. - (i) follows by Corollary (5.3). Concerning (ii) we point out that in this case $\mathcal{L}_{0}(y)=\left(\alpha_{i} y_{i}^{\alpha_{i}-1}\right)_{i=1, \ldots, N}$ and so $\operatorname{Jac} \mathcal{L}_{0}(0)$ is invertible. Hence the claim follows by Theorem 1.1 and Remark 3.7.

In the next corollary we deduced a uniqueness result for nongenerate critical points (see [2] for a similar result)

COROLlary 6.4. - Let us suppose that $P$ is a nondegenerate critical point of $V$. Then there is only one single-peak solution of (1.4) concentrating at $P$

Proof. - By Theorem 1.1 it is enough to prove that $Z$ is a singleton. This follows by Lemma 5.4.

\section{Acknowledgement}

I would like to thank E.N. Dancer for his useful remarks concerning Section 6.

\section{REFERENCES}

[1] A. Ambrosetti, M. Badiale, S. Cingolani, Semiclassical states of nonlinear Schrödinger equations, Arch. Rat. Mech. Anal. 140 (1997) 285-300.

[2] D. Cao, E. Noussair, S. Yan, Existence and uniqueness results on single peaked solutions of a semilinear problem, Ann. Inst. H. Poincaré 15 (1998) 73-111.

[3] E.N. Dancer, On the uniqueness of the positive solution of a singularly perturbed problem, Rocky Mountain J. Math. 25 (1995) 957-975.

[4] M. Del Pino, P.L. Felmer, Local mountain passes for semilinear elliptic problems in unbounded domains, Calc. Var. PDE 149 (1997) 245-265.

[5] M. Del Pino, P.L. Felmer, Semiclassical states of nonlinear Schrödinger equations, J. Funct. Anal. 149 (1997) 245-265.

[6] W.Y. Ding, W.M. Ni, On the existence of positive entire solutions of a semilinear elliptic equation, Arch. Rat. Mech. Anal. 91 (1986) 283-308.

[7] A. Floer, A. Weinstein, Nonspreading wave packets for the cubic Schrödinger equation with a bounded potential, J. Funct. Anal. 69 (1986) 397-408.

[8] M. Grossi, Some results for a class of nonlinear Schrödinger equations, Math. Z. 235 (2000) 687-705.

[9] B. Gidas, W.M. Ni, L. Nirenberg, Symmetry of positive solutions of nonlinear elliptic equations in $\mathcal{R}^{N}$, in: Mathematical Analysis and Applications, Part A, Adv. Math. Suppl. Studies, Vol. 7A, Academic Press, New York, 1981.

[10] D. Gilbarg, N. Trudinger, Elliptic Partial Differential Equations of Second Order, Springer, Berlin, 1977.

[11] M.K. Kwong, Uniqueness of positive solutions of $\Delta u-u+u^{p}=0$ in $\mathcal{R}^{n}$, Arch. Rat. Mech. Anal. 105 (1989) 243-266.

[12] Y.Y. Li, On a singularly perturbed elliptic equation, Adv. Diff. Eqns. 2 (1997) 955-980. 
[13] Lloyd, Degree Theory, Cambridge University Press.

[14] W.M. Ni, I. Takagi, On the shape of least energy solutions to a semilinear Neumann problem, Comm. Pure Math. Appl. 41 (1991) 819-851.

[15] W.M. Ni, J. Wei, On the location and profile of spike-layer solutions to singularly perturbed semilinear Dirichlet problems, Comm. Pure Math. Appl. 48 (1995) 731-768.

[16] Y.G. Oh, Existence of semiclassical bound states of nonlinear Schrödinger equation with potential in the class $(V)_{\alpha}$, Comm. Part. Diff. Eq. 13 (1988) 1499-1519.

[17] P. Rabinowitz, On a class of nonlinear Schrödinger equation, Z. Angew. Math. Phys. 43 (1992) 270-291.

[18] X. Wang, On a concentration of positive bound states of nonlinear Schrödinger equations, Comm. Math. Phys. 153 (1993) 223-243. 